\title{
Bisphosphonate therapy for chronic recurrent multifocal osteomyelitis
}

\author{
Brian Handly • Michael Moore • Gina Creutzberg • \\ Brandt Groh • Timothy Mosher
}

Published online: 23 April 2013

(C) ISS 2013

\section{Discussion}

This patient was diagnosed with chronic multifocal recurrent osteomyelitis of the ankle approximately 4 years prior to the presented images. Multiple treatments were administered, most notably monthly intravenous pamidronate, a therapy that was continued for 17 months, until recrudescent disease prompted a change in therapy. At the time of the presented images, the patient had not received bisphosphonate therapy for 2 years.

Bisphosphonates are a class of medications used primarily in adult patients with decreased bone mineral density. When the drug is incorporated into the hydroxyapatite matrix of nascent bone, osteoclastic activity is inhibited, and as a result, bone mineral density increases. Also used in patients with expansile bone lesions, the decrease in osteoclastic activity serves to slow the progression of enlargement, demonstrating an analgesic effect. Finally, it is thought that bisphosphonates engender a direct anti-inflammatory response by altering the inflammatory cascade [1].

In children, bisphosphonates have been used for the treatment of conditions resulting in low bone mineral density, such as osteogenesis imperfecta [2]. Furthermore, several recent studies have demonstrated the effectiveness of bisphosphonate therapy as a second-line treatment for CRMO-associated pain [3]. Most CRMO treatment regimens consist of daily infusions of IV pamidronate for 1-3 days, repeated every 3 months $[1,3,4]$. Pain relief is usually rapid, within days of

initiation, and resolution of inflammation by MR imaging follows in up to $80 \%$ of patients [2].

Radiologically, the cyclical inhibition of osteoclast activity in the skeletally immature patient gives rise to dense metaphyseal bands migrating longitudinally from the zone of provisional calcification into the diaphysis, as previously described [5, 6]. Additionally, undertubulation of the affected areas, as well a "bone within a bone" appearance can be seen [7]. The presence of metaphyseal bands engenders differential considerations such as lead poisoning and hypervitaminosis $\mathrm{D}$, as well as causes of standard growth recovery lines (times of malnutrition, severe illness, etc.). As none of the causes of metaphyseal bands are common, the interpreting radiologist may be inspired to suggest further testing when confronted with this unusual finding. However, the recognition of focal undertubulation (most striking at the distal femur in this case) helps cinch the diagnosis. Recognition of these findings can be clinically important, as the abrupt change of bone mineral density at the dense bands has been implicated as a stress riser, similar to the interface between a prosthesis and native bone [8]. The potential increase in fracture risk presented by these bands may prompt a change in the duration of therapy.

Conflicts of interest The authors declare that they have no conflicts of interest.

\section{References}

The case presentation can be found at doi:10.1007/s00256-013-1614-7

B. Handly $(\bowtie) \cdot$ M. Moore $\cdot$ G. Creutzberg $\cdot$ B. Groh $\cdot$ T. Mosher Milton S. Hershey Medical Center, 500 University Drive, Hershey, PA 17033, USA

e-mail: handly@gmail.com

1. Simm P, Allen R, Zacharin M. Bisphosphonate treatment in chronic recurrent multifocal osteomyelitis. J Pediatr. 2008;152:571-5.

2. Ferguson P, Sandu M. Current understanding of the pathogenesis and management of chronic recurrent multifocal osteomyelitis. Curr Rheumatol Rep. 2012;14(2):130-41. 
3. Twitt M, Laxer R. Clinical care of children with sterile bone inflammation. Curr Opin Rheumatol. 2011;23(5):424-31.

4. Hospach T, Langendoerfer M, von Kalle T, et al. Spinal involvement in chronic recurrent multifocal osteomyelitis (CRMO) in childhood and effect of pamidronate. Eur J Pediatr. 2010;169: 1105-11.

5. Grissom L, Harcke H. Radiographic features of bisphosphonate therapy in pediatric patients. Pediatr Radiol. 2003;33: 226-9.
6. Sidhu H, Venkatanarasimha N, Bhatnagar G, et al. Imaging features of therapeutic drug-induced musculoskeletal abnormalities. Radiographics. 2012;32:105-27.

7. van Meerten E, Kroon H, Papapoulos S. Epi- and metaphyseal changes in children caused by administration of bisphosphonates. Radiology. 1992;184:249-54.

8. Harcke H, Stevenson K, Kecskemethy H, et al. Fracture after bisphosphonate treatment in children with cerebral palsy: the role of stress risers. Pediatr Radiol. 2012;42:76-81. 